\title{
Behavioral Consequences of Bicuculline Injection in the Subthalamic Nucleus and the Zona Incerta in Rat
}

\author{
Céline Périer, Léon Tremblay, Jean Féger, and Etienne C. Hirsch \\ Institut National de la Santé et de la Recherche Médicale U289, Experimental Neurology and Therapeutics, Hôpital de la \\ Salpêtrière, 75013 Paris, France
}

\begin{abstract}
The subthalamic nucleus (STN) plays a crucial role in basal ganglia functions and has been shown to be hyperactive in parkinsonian syndromes. The zona incerta (ZI), located dorsally to the STN, is also reported to be overactive after nigrostriatal denervation. In this study, we examined the behavioral consequences of an increased activity of the STN or the ZI in awake, freely moving rats. Unilateral microinjections of a $\mathrm{GABA}_{\mathrm{A}}$ receptor antagonist (bicuculline; 25, 50, and $100 \mu \mathrm{g} / \mu \mathrm{l}$ ) were performed in the STN or in the ZI of rats, and locomotor activity, spontaneous behaviors, and the occurrence of abnormal movements were quantified. Microinjection of bicuculline (50 and 100 $\mu \mathrm{g} / \mu \mathrm{l}$ ) into the STN did not modify spontaneous locomotor activity, whereas it induced an increase in locomotion when injected into the ZI. Furthermore, when injected into the STN or
\end{abstract}

Within the basal ganglia, the subthalamic nucleus (STN) plays a key role in the control of motor functions. Several lines of evidence suggest that in humans, lesion of the STN provokes the appearance of hemiballism characterized by gross involuntary movements of the limbs on one side of the body (Martin, 1927). Other studies, in monkey, have demonstrated a direct relationship between hemiballism and discrete lesions of the STN destroying only 20\% of the structure (Whittier and Mettler, 1949; Carpenter et al., 1950; Hammond et al., 1979; Hamada and DeLong, 1992). In parkinsonism, on the other hand, it has been shown that the hyperactivity of the STN is involved in the motor deficit of the disease because neurosurgical lesions or high-frequency stimulation of the STN in parkinsonian patients (Limousin et al., 1995) and in animals with experimentally induced parkinsonism (Bergman et al., 1990; Benazzouz et al., 1993; Guridi and Obeso, 1997) greatly improve the motor signs of the disease. Thus, in some movement disorders, STN activity may determine which clinical sign is observed: a hyperactive state being involved in akinesia, and a hypoactive state in hyperkinesia. Moreover, a recent study has shown that, in monkeys rendered parkinsonian by MPTP intoxication, hypoactivity induced by inactivation of discrete regions within the STN may ameliorate parkinsonian motor signs, whereas hypoactivity in nonmotor territories may induce circling

Received May 7, 2002; revised July 9, 2002; accepted July 10, 2002.

This work was supported by Institut National de la Santé et de la Recherche Médicale (INSERM) and the National Parkinson Foundation (Miami, FL). C.P. was supported by a fellowship grant from the Ministère de l'Education Nationale et de la Recherche and from the Fondation pour la Recherche Medicale (France). We thank M. H. Thiebot (INSERM U288) for providing the behavioral testing cage.

Correspondence should be addressed to Etienne C. Hirsch, Institut National de la Santé et de la Recherche Médicale U289, Experimental Neurology and Therapeutics, Hôpital de la Salpêtrière, 47 Boulevard de l'Hôpital, 75013 Paris, France. E-mail: hirsch@ccr.jussieu.fr.

Copyright (ㄷ) 2002 Society for Neuroscience $0270-6474 / 02 / 228711-09 \$ 15.00 / 0$
$\mathrm{ZI}$, these same doses of bicuculline produced changes in spontaneous behaviors (sniffing and grooming decreased whereas chewing and rearing increased) and the appearance of abnormal movements directed contralaterally to the injection side. Application of a lower dose of bicuculline $(25 \mathrm{ng} / \mu \mathrm{l})$ in the STN or ZI did not modify behavior. This study suggests that the subthalamic region including the $\mathrm{ZI}$, and not the STN per se, might be involved in the induction of abnormal movements. In addition, these data suggest that the hyperactivity of neurons in this region may have different consequences in the normal state and in the pathological state.

Key words: bicuculline; zona incerta; subthalamic nucleus; behavior; rat; microinjection

and behavioral abnormalities (Baron et al., 2002), suggesting a more subtle involvement of the STN in the pathophysiology of movement disorders.

We recently demonstrated, using metabolic and electrophysiologic measurements, that the zona incerta (ZI), a structure dorsal to the STN in the rat, is also hyperactive after a lesion of the dopaminergic neurons in rats (Périer et al., 2000). The neurons of the ZI showed an increase in the mean discharge rate without changes in the discharge pattern, this increase being more pronounced in the dorsolateral part of the structure. From a physiological point of view, the ZI is considered to be a component of the subthalamic locomotor region and is involved in motor function. Indeed, increases in locomotor activity have been observed after electrical stimulation and injection of pharmacological agents into the ZI (Grossman, 1958; Milner and Mogenson, 1988). Furthermore, the ZI is interconnected, along with other brain regions, with cerebral structures involved in motor functions, such as the internal pallidum, the superior colliculus, the pedunculopontine nucleus, and the somatosensory cortex (Roger and Cadusseau, 1985; Romanowski et al., 1985; Kolmac et al., 1998). Thus, a hyperactivity of the ZI could also participate in the production of parkinsonian symptoms.

The main goal of our study was to determine whether a local hyperactivity of STN and ZI neurons in the normal rat could induce parkinsonian signs. A local and reversible neuronal hyperactivity was therefore induced by a local, unilateral microinjection of the $\mathrm{GABA}_{\mathrm{A}}$ receptor antagonist bicuculline in these two structures. The concentrations of bicuculline used in our study were close to those reported to induce a significant increase in the mean spontaneous neuronal discharge rate (Féger et al., 1989; Robledo and Féger, 1990). Our study of the behavioral consequences of these microinjections included an analysis of (1) 
locomotor activity, (2) spontaneous behaviors, and (3) abnormal movements.

\section{MATERIALS AND METHODS}

Animals. Male Sprague Dawley rats weighing 280-350 gm (CERJ, Le Genest St-Isle, France) were housed on a $12 \mathrm{hr}$ light/dark cycle under constant temperature and humidity conditions and given ad libitum access to food and water. All studies were performed in accordance with the Declaration of Helsinki and the Guide for the Care and Use of Laboratory Animals adopted and promulgated by National Institutes of Health. The number of animals used was distributed as follows: bicuculline microinjection into the STN at $25 \mathrm{ng} / \mu \mathrm{l}(n=8), 50 \mathrm{ng} / \mu \mathrm{l}(n=7)$, and $100 \mathrm{ng} / \mu \mathrm{l}(n=7)$, and into the $\mathrm{ZI}$ at $25 \mathrm{ng} / \mu \mathrm{l}(n=13), 50 \mathrm{ng} / \mu \mathrm{l}(n=$ $18)$, and $100 \mathrm{ng} / \mu \mathrm{l}(n=11)$.

Implantation of intracerebral guide cannulas. Rats were prepared surgically $4-5 \mathrm{~d}$ before experimental testing. The rats were anesthetized with $0.2 \mathrm{ml} / 100 \mathrm{gm}$ (intramuscularly) of a solution containing $50 \mathrm{mg} / \mathrm{ml}$ of ketamine and $80 \mathrm{mg} / \mathrm{ml}$ of xylazine, and a chronic intracerebral guide cannula was then stereotaxically implanted on one side of the brain. Guide cannulas were positioned $2 \mathrm{~mm}$ dorsally to the target structures using the rat brain atlas of Paxinos and Watson (1986): for the ZI, anteroposterior (AP) -3.2 to -3.8 posterior to the bregma, mediodorsal (MD) 1.7-2.6, 6.3-7.8 mm below the dura; for the STN, AP -3.2 to -3.5 posterior to the bregma, MD 2.2-2.4, 7.3-7.8 $\mathrm{mm}$ below the dura. Guide cannulas were inserted into holes drilled in the skull and were fixed in place with dental resin. Stainless steel stylettes were inserted into guides to keep them clean before and between injections.

Microinjections. Bicuculline methiodide (Sigma, St. Quentin Fallavier, France) was dissolved in saline at three concentrations: 25, 50, or 100 $\mathrm{ng} / \mu \mathrm{l}$.

Injections were performed over a period of $2 \mathrm{~min}$ at a rate of 0.1 $\mu \mathrm{l} / \mathrm{min}$ in awake, freely moving rats. Drug solutions were injected via an acute injection cannula (28 gauge) inserted into the guide cannula (22 gauge). The injection cannula, which extended $2 \mathrm{~mm}$ beyond the tip of the guide cannula, was connected by a catheter to a microsyringe (10 $\mu \mathrm{l}$ airtight, Hamilton) mounted on a motorized driver. The injection cannula was left in place for $1 \mathrm{~min}$ after each injection before removal.

All testing was done between 8:00 A.M. and 3:00 P.M. in an isolated room. After a habituation period, each rat was given five injections at minimum intervals of $24 \mathrm{hr}$. Bicuculline injection were preceded and followed by a $0.9 \%$ saline injection. Each animal received two unilateral bicuculline injections in the ZI or the STN of either 25,50 , or $100 \mathrm{ng} / \mu \mathrm{l}$ (Fig. 1).

Behavioral analysis. Behavior was observed and recorded with a camera placed above the cage, immediately after each microinjection for a period of $30 \mathrm{~min}$. During this observation period, the animals were placed in a $37 \times 37 \mathrm{~cm}$ clear plastic cage. Motor behavior was analyzed using Vigiprimate software (Viewpoint, Lyon, France), which allows the motor activity of the animals to be finely analyzed. This software uses a window discriminator to distinguish three levels of movement: (1) immobility, or absence of movement, which may represent a measurement of akinesia or freezing; (2) movement without displacement, during which rats display behaviors such as grooming, sniffing, etc., and (3) displacement within the cage.

To further characterize spontaneous behaviors and the occurrence of abnormal movements, we counted and quantified each behavior or abnormal movements by observation on videotape. Our study included the analysis of four kinds of behavior, namely sniffing, grooming, chewing, and rearing and five types of abnormal movement, namely head movements, axial torsion, jumping, and limb movements (flexions/extensions and extension movements). The forelimb movements consisted in successive alternate flexion and extension when animals were on the floor and in repetitive striking, extension movements of the forelimb during rearing.

The intensity of various behaviors or movements (sniffing, grooming, chewing, flexions/extensions, and extension movements) was assessed according to a modified version of the scoring scale of Creese and Iversen (1974): $0=$ duration $<5 \mathrm{sec}$; $1=$ duration of $5-15 \mathrm{sec} ; 2=$ duration of $15-40 \mathrm{sec} ; 3=$ duration of $40-80 \mathrm{sec} ; 4=$ duration $>80 \mathrm{sec}$. Other kinds of behavior or movement such as rearing, head movements, axial torsion, and jumping were quantified as the number of occurrences over time. Measurements were made throughout the $30 \mathrm{~min}$ period after microinjection.

Electrophysiology. After the behavioral observation period, electrophysiological recordings were performed on some of the animals to verify

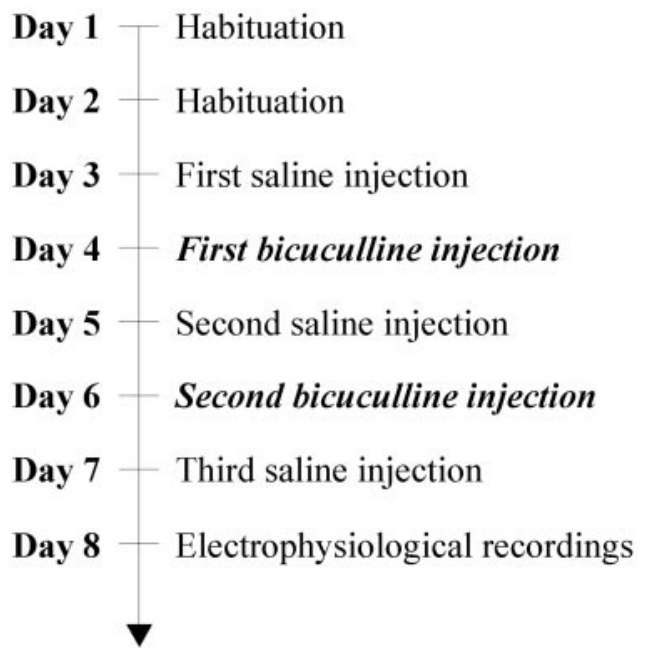

Figure 1. Experimental design of the behavioral analysis. After surgery, all rats were kept in their cages with ad libitum access to food and water and were regularly habituated to the experimenter. The two first sessions were conducted to habituate the animals to the cages used for behavioral analysis. Three saline injections alternated with the bicuculline injections to ascertain that the behaviors observed after bicuculline injection were specific to the injection and were not caused by a possible long-term residual effect of the injection or habituation to the test environment. In some cases, electrophysiological recordings were made after the third saline injection.

that the cannula did not produce a lesion. Unit activity was recorded using Teflon- and epoxylite-insulated tungsten microelectrodes (stem of $76 \mu \mathrm{m}$ outer diameter, tapered down over $0.5 \mathrm{~mm}$ to exposed tips of $1.5-5.0 \mu \mathrm{m}$ in diameter and 5-25 $\mu \mathrm{m}$ in length) with an impedance of $1.5-6.0 \mathrm{M} \Omega$ Briefly, a cannula (30 gauge) containing the microelectrode was inserted into the guide cannula, and extracellular single-unit recordings were made. The signals were amplified (DAM5 WPI), displayed on a storage oscilloscope and recorded on a computer. Single-unit activity was processed using a window discriminator providing pulses that were sent to the input of an integrator or processed using a computer (Interface micro 1401 and Spike-2 software; Cambridge Electronic Design, Cambridge, UK).

Histology. After testing, the animals were killed with an overdose of pentobarbital, and the brains were removed and frozen in isopentane. Serial sections (50- $\mu \mathrm{m}$-thick) containing evidence of the needle tracks were mounted directly onto glass slides and stained with cresyl violet. Staining for gliosis along the needle tracks allowed the location of the deepest point of penetration to be identified. This point was taken as the center of the injection site (Fig. 2) and marked on coronal sections modified from the atlas of Paxinos and Watson (1986) (Fig. 3). Animals in which the injection sites were not located in the structures of interest were eliminated from data analysis. Most of these injections were located in the thalamus and used to control the absence of diff usion toward the thalamus (Fig. 3). None of these injections induced behavioral effects.

Statistical analysis. Data are presented as mean \pm SEM. For a given injection site (STN or ZI), comparisons between the different doses of bicuculline were made by one-way ANOVA or, in the event of a failure in the test of normality, by one-way ANOVA on ranks (Kruskal-Wallis test). When ANOVA showed a statistically significant difference, it was followed by Fisher's LSD post hoc analysis or, when the Kruskal-Wallis test was used, by Dunn's test. To determine whether there was any interaction between injection site and bicuculline dose, a two-way ANOVA followed by a Fisher's LSD post hoc analysis was used (Sigmastat software).

\section{RESULTS}

\section{Validation of the behavioral experimental design}

Preliminary studies showed that after two sessions of habituation the behavior of the animals was stable, indicating that the rats were accustomed to their test environment. 


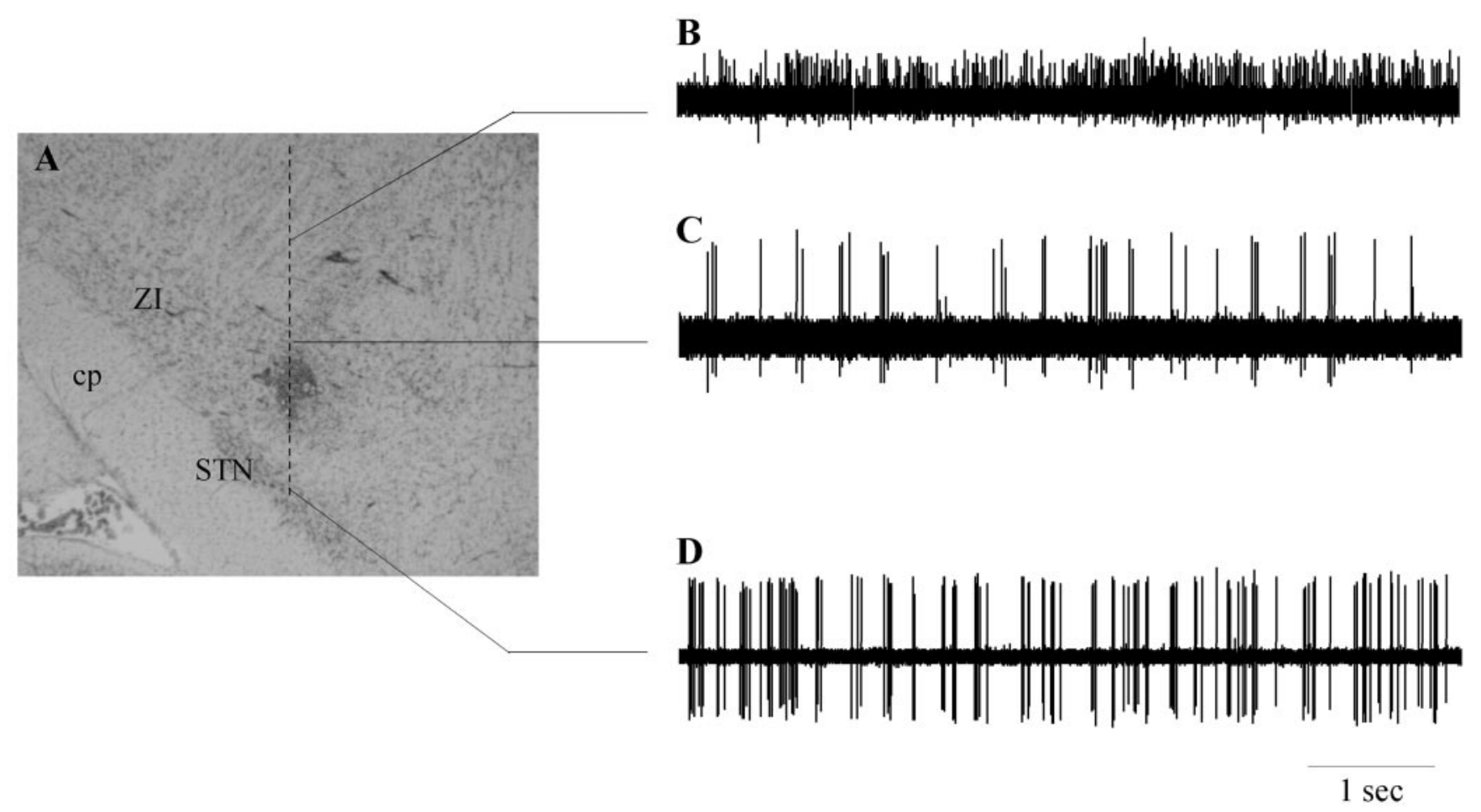

Figure 2. Localization of the center of injection. A, Photomicrograph showing a coronal section stained with cresyl violet. The injected structure is easily visible as a gliosis seen at the center of the injection. The dotted line represents the track of the metallic electrode used to record neurons. Multiple neuronal activity was recorded $(B)$ in the thalamus dorsal to the ZI, and single neuronal activity was recorded $(C)$ in the ZI and $(D)$ in the STN. $c p$, Cerebral peduncle; STN, subthalamic nucleus; ZI, zona incerta.

A

\section{B}
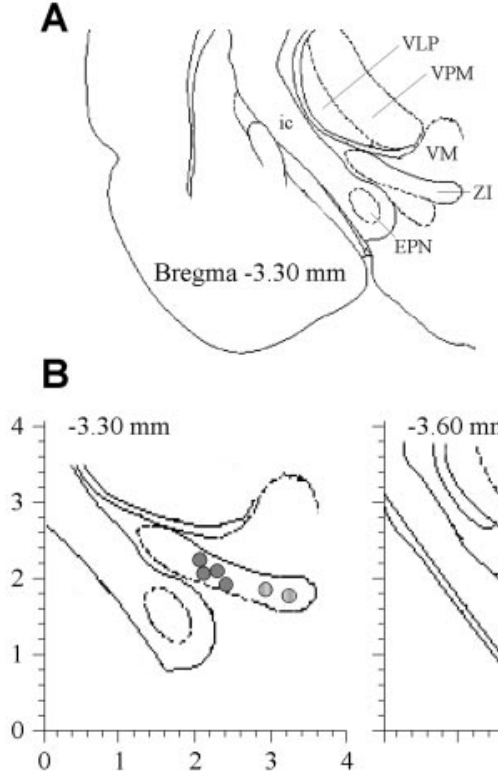
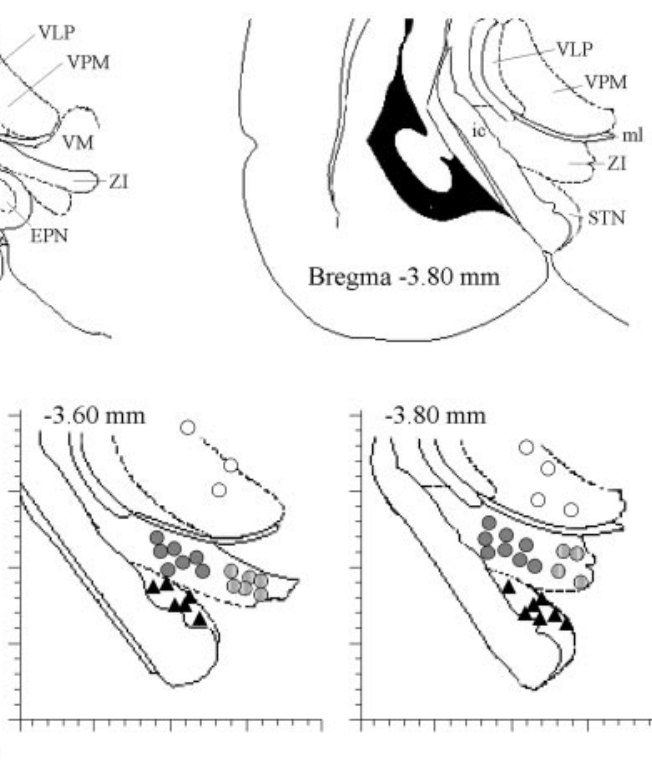
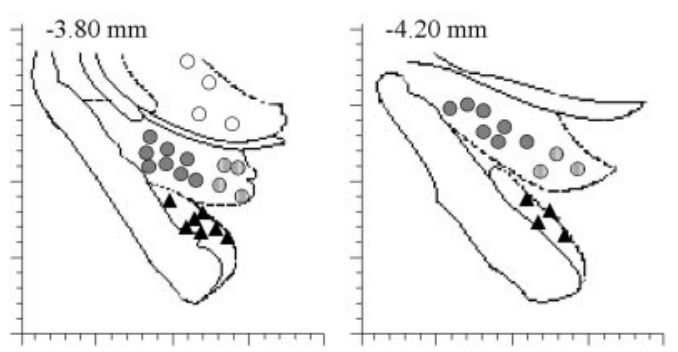
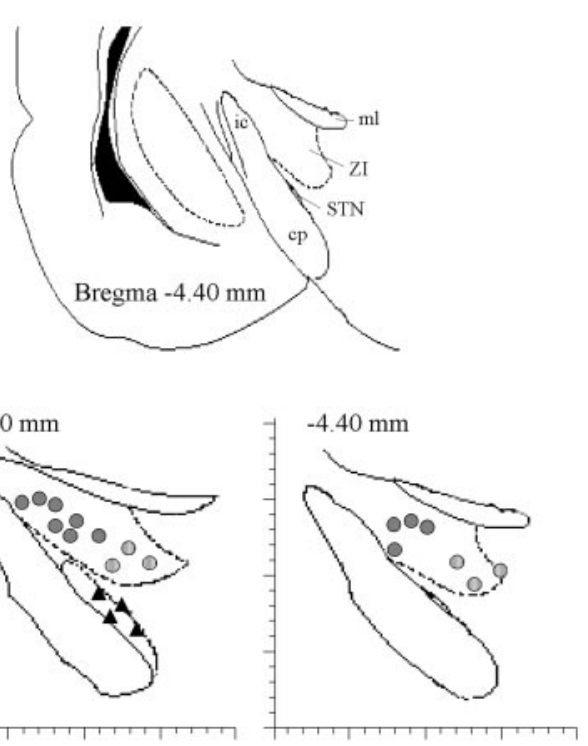

Figure 3. Coronal sections depicting areas of microinjections into the STN, ZI, and dorsal to the subthalamic region. For histology, coronal sections were cut in the plane corresponding to the atlas of Paxinos and Watson (1986), from which these drawings were derived $(A)$. $B$, Magnification of the subthalamic region showing the localization of the microinjection site. Black triangle areas designate the regions of the STN. Hatched areas designate medial ZI sites of injection. Gray areas designate lateral ZI injection sites. White areas represent the injection sites located dorsally to the subthalamic region. $C P$, Cerebral peduncle; $E P N$, entopeduncular nucleus; $i c$, internal capsule; $m l$, medial lemniscus; $S T N$, subthalamic nucleus; $V M$, ventromedial nucleus of the thalamus; VPL, ventral posterolateral thalamic nucleus; VPM, ventral posteromedial thalamic nucleus; $Z I$, zona incerta.

After the first saline injection, the rats exhibited the same behavior as those that had not been injected (data not shown). Each rat had a specific spontaneous behavior, characterized by sniffing, grooming, chewing, and rearing, and no abnormal move- ments were induced by saline microinjections. During the $30 \mathrm{~min}$ session, a decrease in the behavioral score was observed for sniffing, grooming, chewing, and rearing (Fig. 4). No differences were observed whether saline injections were made into the $\mathrm{ZI}$ or 


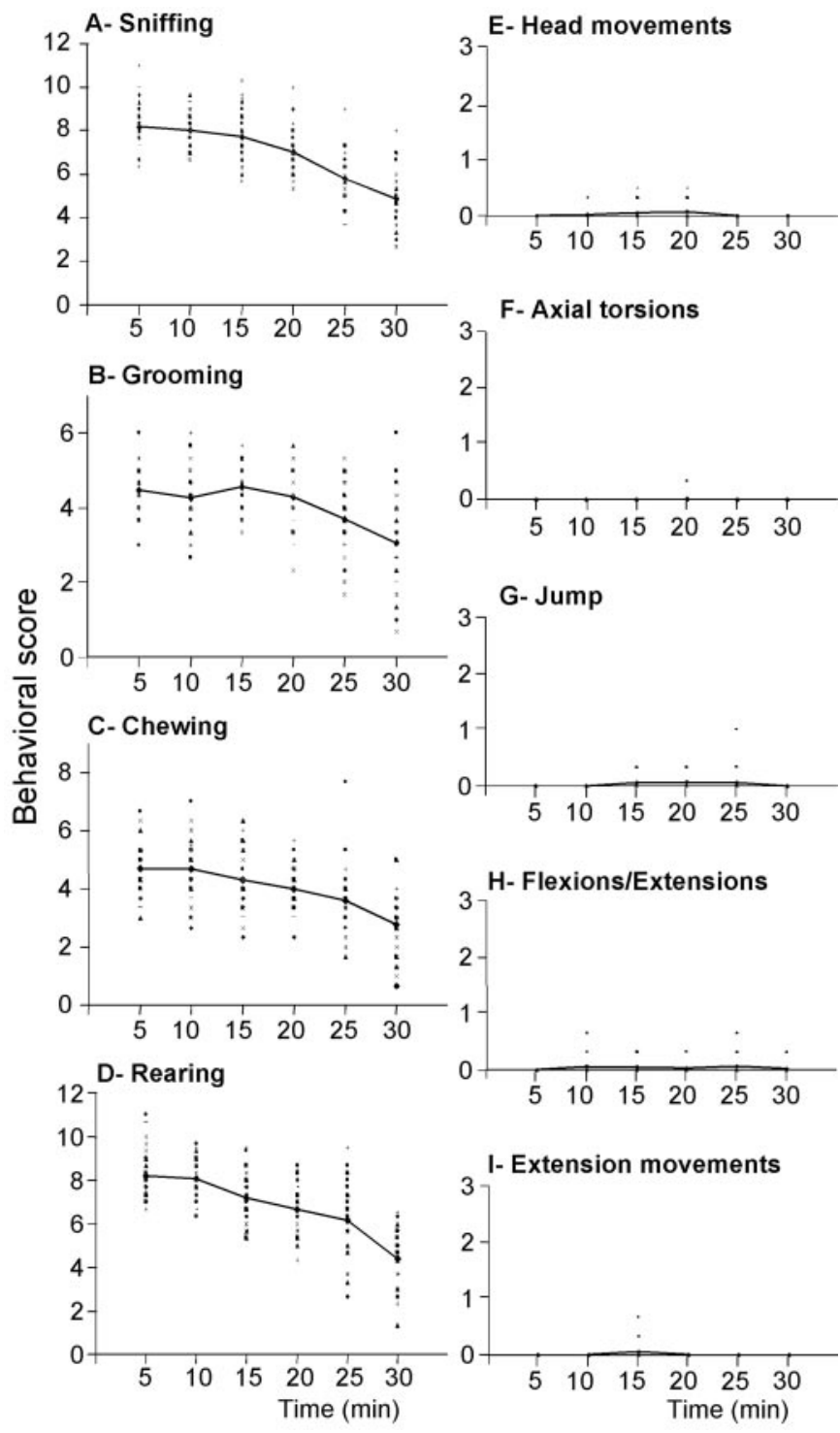

Figure 4. Behavioral scores analyzed during $5 \mathrm{~min}$ periods for $30 \mathrm{~min}$ after a saline injection, showing the variability between rats. Horizontal line represents group mean values \pm SEM of scores for the various behaviors after saline microinjection in the STN. Each dot represents the behavioral score of an individual rat. Behavioral scores were made for 5 min at the indicated time points. $T=0$ corresponds to the microinjection time.

the STN. After five consecutive saline microinjections into the same structure, we did not observe any behavioral differences between the first and the fifth session, indicating that five penetrations of the cannula did not induce a lesion capable of modifying the rats' behaviors or movements. The absence of lesion was also demonstrated by the presence of active neurons in 10 rats of 13 recorded rats, detected by extracellular electrophysiological recordings after the five microinjections (Fig. 2). The mean discharge rate was $9.92 \pm 2.05$ for the STN neurons and $4.82 \pm 1.72$ for the ZI neurons. These values were close to those previously reported (Périer et al., 2000; Vila et al., 2000).

Observations of spontaneous behavior in rats receiving saline injections, as well as in noninjected rats (data not shown), evidenced a considerable degree of behavioral variability between animals (Fig. 4). This observation led us to choose a behavioral experimental design in which each animal was its own control to analyze the effect of bicuculline injection. This also allowed us to keep the number of animals used to a minimum. Thus, to test whether bicuculline injection had a residual effect, behaviors were compared for saline microinjections made before and after the two bicuculline injections. Except for sniffing behavior, which differed between the first saline injection and the other two, no differences were found between the three saline microinjections for any of the studied behaviors (Table 1), suggesting that there was no residual behavior after bicuculline injection.

Moreover, to assess that the observed effects were not caused by a diff usion toward the thalamus, we realized some injections in the thalamus (Fig. 3). Seven injections were made for the two highest concentrations of bicuculline, $50 \mathrm{ng} / \mu \mathrm{l}(n=7)$ and 100 $\mathrm{ng} / \mu \mathrm{l}(n=6)$. No injections of bicuculline at $25 \mathrm{ng} / \mu \mathrm{l}$ were realized, because no difference in spontaneous behaviors were observed for these two highest concentrations compared with saline injections (Table 2). No appearance of abnormal movements was observed after microinjection of bicuculline into the thalamus.

\section{Immobility and locomotor effects of bicuculline microinjection into the STN and the ZI}

Unilateral microinjection of bicuculline, at any of the three concentrations, did not induce significant changes in the immobility score when injected into the STN or the ZI.

Microinjection of bicuculline did not induce significant changes in locomotor activity when microinjected into the STN but, at the highest two doses, induced a marked increase in locomotor activity when microinjected into the ZI (Fig. 5). Thus, at the lowest concentration $(25 \mathrm{ng} / \mu \mathrm{l})$, no increase in spontaneous locomotor activity was observed when injected into either the STN or the ZI. In contrast, when bicuculline was injected into the ZI at a concentration of 50 or $100 \mathrm{ng} / \mu \mathrm{l}$, a significant increase in spontaneous locomotor activity was observed. This increased level of motor activity remained constant for up to $20 \mathrm{~min}$ after injection, thereafter returning to the control level. For the middle (50 $\mathrm{ng} / \mu \mathrm{l})$ and highest $(100 \mathrm{ng} / \mu \mathrm{l})$ concentrations of bicuculline injected into the STN, no increase in locomotion was found, but the rats showed a greater variation in their behavior.

\section{Behavioral and movement effects of bicuculline microinjection into the STN and the ZI}

At no time during the observation period were catalepsy or tremor observed after bicuculline microinjection into the STN or the $\mathrm{ZI}$.

The number and intensity of sniffing and grooming behaviors normally displayed by the animals decreased after bicuculline microinjection into the STN and ZI, whereas those of chewing and rearing increased (Fig. 6). Microinjection of bicuculline into the STN or the ZI resulted in the appearance of axial torsions and head movements toward the side contralateral to the injection. These abnormal movements were accompanied by jumping and limb movements (flexions/extensions and extension movements). Jumping and axial torsions were sometimes followed by falls.

In the STN, the lowest concentration $(25 \mathrm{ng} / \mu \mathrm{l})$ of bicuculline used induced only an increase in the frequency of rearing. The same concentration injected into the ZI provoked an increase in the frequency of chewing and of rearing. An injection of bicuculline at a higher concentration (50 and $100 \mathrm{ng} / \mu \mathrm{l})$ into the STN or the ZI induced (1) a decrease in sniffing and grooming, (2) an 
Table 1. Comparison of spontaneous behaviors after three saline injections alternating with two bicuculline injections (for details, see Materials and Methods)

\begin{tabular}{|c|c|c|c|c|}
\hline & Sniffing & Grooming & Chewing & Rearing \\
\hline First saline injection & $51.81 \pm 5.63$ & $26.69 \pm 3.73$ & $25.57 \pm 3.63$ & $43.57 \pm 6.44$ \\
\hline Second saline injection & $36.54 \pm 6.36^{*}$ & $24.06 \pm 5.22$ & $23.36 \pm 4.94$ & $39.75 \pm 8.55$ \\
\hline Third saline injection & $35.59 \pm 7.55^{*}$ & $23.03 \pm 5.84$ & $22.03 \pm 6.55$ & $39.31 \pm 7.7$ \\
\hline
\end{tabular}

Values represent group mean values \pm SEM of scores for each behavior.

$* p<0.05$.

Table 2. Behavioral effects of bicuculline injection into the thalamus

\begin{tabular}{|c|c|c|c|c|}
\hline & Sniffing & Grooming & Chewing & Rearing \\
\hline Bicuculline $50 \mathrm{ng} / \mu \mathrm{l}$ & $41.31 \pm 6.51$ & $24.59 \pm 5.59$ & $23.65 \pm 5.04$ & $40.87 \pm 7.56$ \\
\hline Bicuculline $100 \mathrm{ng} / \mu \mathrm{l}$ & $43.78 \pm 8.56$ & $22.58 \pm 4.68$ & $28.76 \pm 7.23$ & $38.54 \pm 6.57$ \\
\hline
\end{tabular}

Values represent group mean values \pm SEM of scores for each behavior on a 30 min session.

Subthalamic nucleus
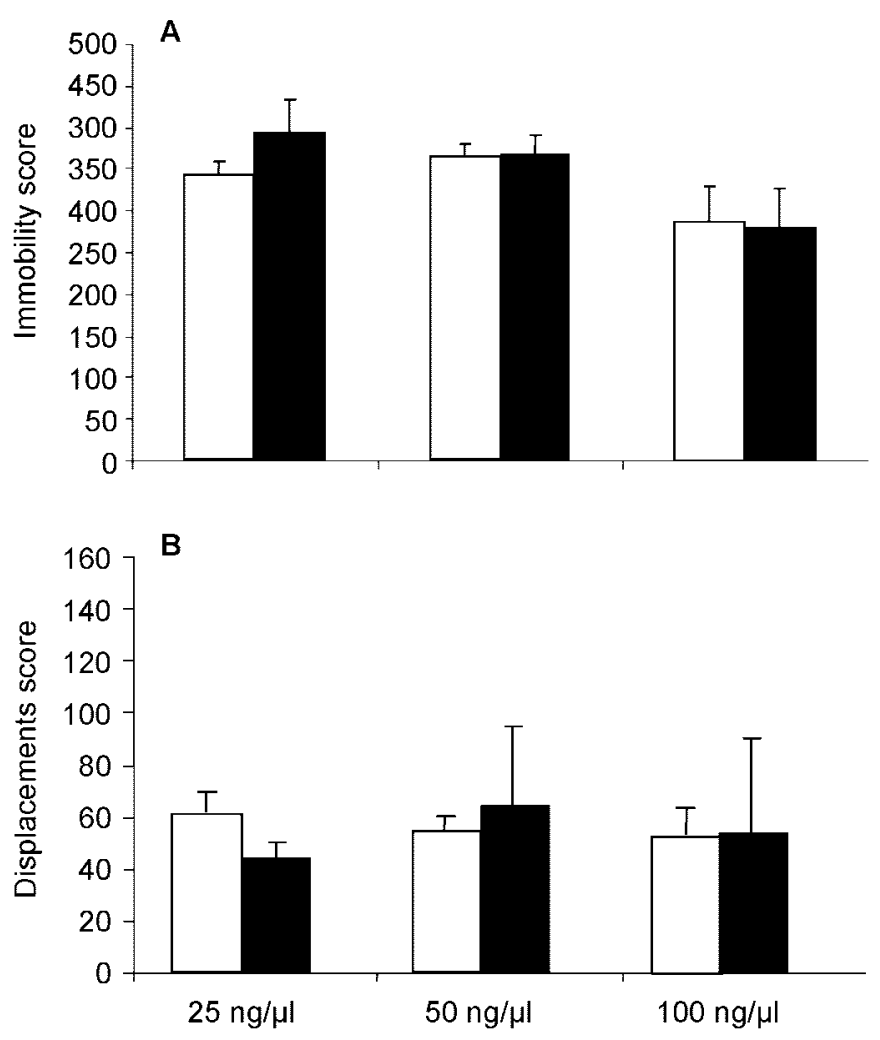

Zona Incerta
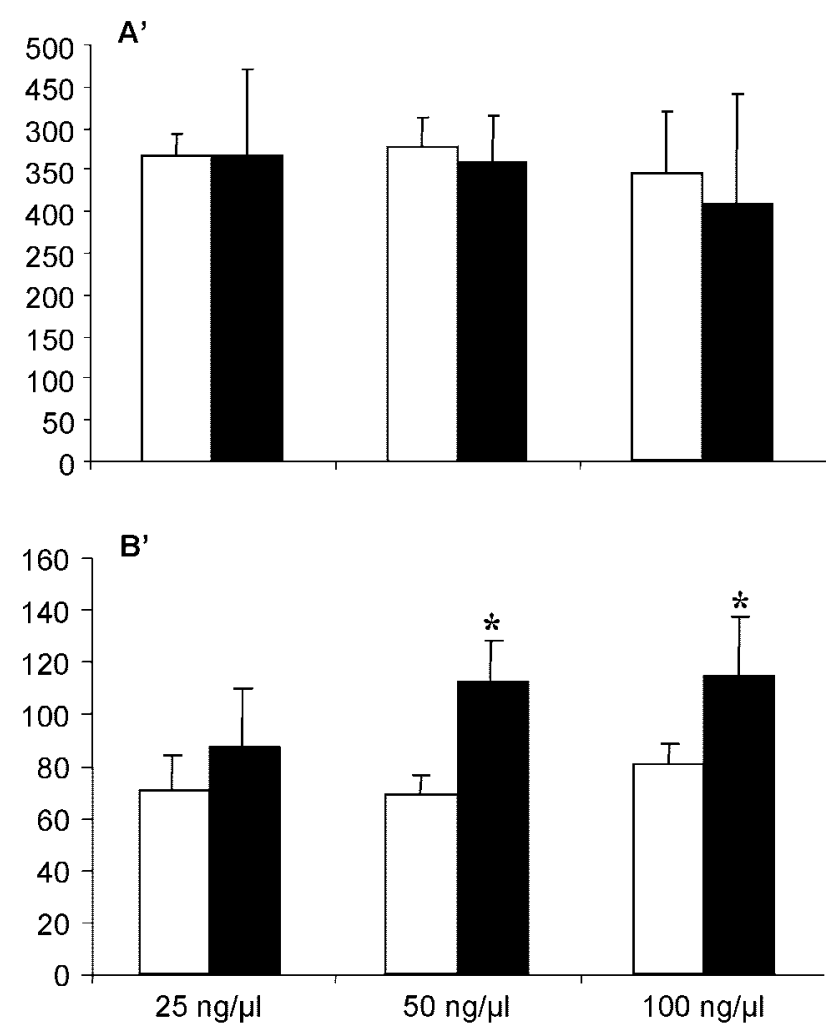

Figure 5. Immobility and locomotor activity after bicuculline injection into the STN ( first column) and the ZI (second column). Values represent group mean values \pm SEM of immobility score $\left(A, A^{\prime}\right)$ or displacement score $\left(B, B^{\prime}\right)$ determined by the Vigiprimate software. This software permits us to distinguish, with a window discriminator, (1) immobility or absence of movement, which may represent a measurement of akinesia or freezing, and (2) displacement within the cage. Bicuculline (black bars) was injected in separate groups of rats into the STN or ZI at three concentrations. $A$, $B$, Microinjection of bicuculline at $25 \mathrm{ng} / \mu \mathrm{l}(n=8), 50 \mathrm{ng} / \mu \mathrm{l}(n=7)$, and $100 \mathrm{ng} / \mu \mathrm{l}(n=7)$ into the STN. $A^{\prime}, B^{\prime}$, Microinjection of bicuculline at $25 \mathrm{ng} / \mu \mathrm{l}$ $(n=13), 50 \mathrm{ng} / \mu \mathrm{l}(n=18)$, and $100 \mathrm{ng} / \mu \mathrm{l}(n=11)$ into the ZI. Asterisks indicate significant difference from respective control values obtained after a saline injection (white bars) (ANOVA and Fisher's post hoc analysis; * $p<0.05$ ).

increase in chewing and rearing, and (3) abnormal movements. With these two higher concentrations, the intensity of the abnormal movements was always less when the injection was made into the ZI than into the STN (Fig. 6).

Modification of each behavior (sniffing, grooming, chewing, and rearing) appeared at the onset of the bicuculline microinjection and continued even in the presence of abnormal movements, which appeared between 10 and 15 min after injection into the STN but between 15 and 25 min after injection into the ZI.

\section{Site variation effect in the STN and in the ZI}

No difference in effect could be evidenced between the different injection sites in the STN.

When the different injection sites in the ZI were compared, the 

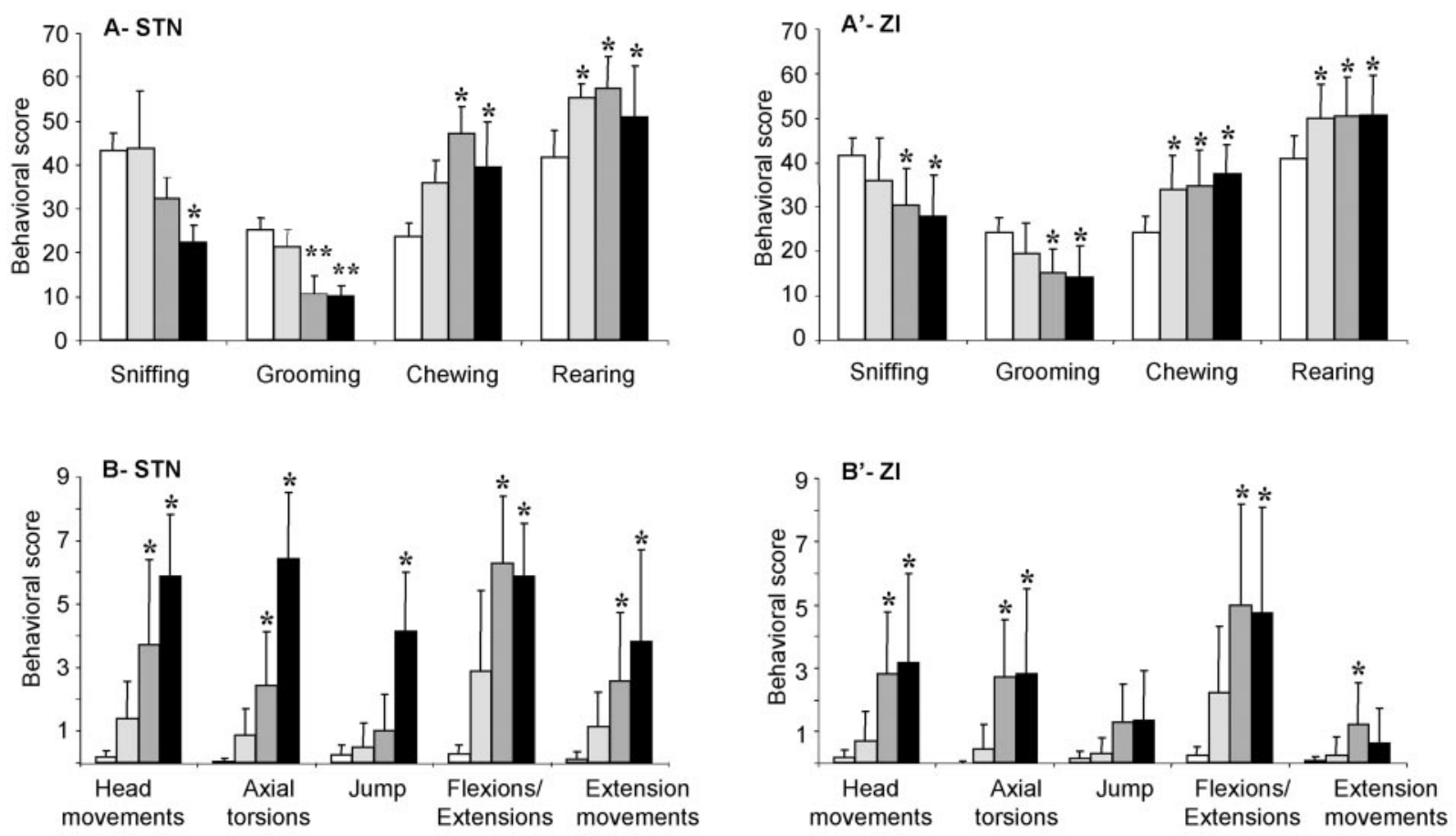

Figure 6. Behavioral effects of bicuculline injection into the STN and the ZI. Values represent group mean values \pm SEM of the different behavioral scores on a $30 \mathrm{~min}$ session. White bars, Saline injections; from light to dark gray: 25, 50, and $100 \mathrm{ng} / \mu \mathrm{l}$ of bicuculline. $A$, Behavioral changes after bicuculline microinjection into the STN at $25 \mathrm{ng} / \mu \mathrm{l}(n=8), 50 \mathrm{ng} / \mu \mathrm{l}(n=7)$, and $100 \mathrm{ng} / \mu \mathrm{l}(n=7)$. $A^{\prime}$, Behavioral changes after bicuculline microinjection into the ZI at $25 \mathrm{ng} / \mu \mathrm{l}(n=13), 50 \mathrm{ng} / \mu \mathrm{l}(n=18)$, and $100 \mathrm{ng} / \mu \mathrm{l}(n=11)$. $B$, Production of abnormal movements after bicuculline microinjection into the STN and $\left(B^{\prime}\right)$ into the ZI at three concentrations. Asterisks indicate significant difference with saline injection (ANOVA and Fisher's post hoc analysis; * $\left.p<0.05,{ }^{*} p<0.01\right)$.

behavioral changes caused by bicuculline injection were found to be more pronounced in the lateral ZI than in the medial ZI (Fig. 7). Indeed, in most cases, there was no difference between control injections and injections of bicuculline in the medial ZI (Fig. 7).

\section{DISCUSSION}

As might be expected, a local hyperactivity of STN neurons in normal rats induced a diminution of behaviors such as sniffing and grooming. However, no changes in the immobility score, which may represent an index of hypokinesia, were observed after bicuculline microinjection. This study also demonstrates that an unilateral application of a $\mathrm{GABA}_{\mathrm{A}}$ antagonist (bicuculline) into the rat STN did not modify the spontaneous locomotor activity, whereas it induced an increase of locomotion when injected into the ZI. Moreover, bicuculline microinjection into both the STN and the ZI induced abnormal movements, such as jumping, axial torsion, and abnormal head and limb movements. The intensity of these abnormal movements was greater when the microinjection was in the STN than in the ZI taken as a whole, and their onset was also more rapid after STN injection. Nevertheless, few differences were found between the STN and the lateral ZI, suggesting that they both play a role in the genesis of these movements. Finally, the decrease in sniffing and grooming preceded the appearance of abnormal movements and did not stop when these movements appeared, suggesting that the decrease of these behaviors and the occurrence of abnormal movements may be the consequence of different mechanisms.

One potential difficulty in interpreting data on intracranial microinjections of pharmacological agents in a given structure is that the observed effects may be caused by drug diffusion to adjacent structures. However, several lines of evidence suggest that this was not the case in our study. First, a diffusion into the thalamus may be ruled out because injections into the thalamus did not induce the behavioral changes observed after injections into the STN or the ZI. Second, a diffusion into the hypothalamus can also be ruled out because no behavioral effects were observed after microinjection into the medial ZI. Finally, it is unlikely that the drug could have diff used from the ZI to the STN because different effects were observed depending on whether the injections were made into the STN or the ZI: greater locomotor activity was observed after activation of the ZI than the STN. Furthermore, the rapid onset and short latency of the occurrence of different behaviors after microinjections in the STN or in the ZI argues against drug diffusion being responsible for the observed effects. Nevertheless, the time between the microinjection and the induction of abnormal movements was always shorter when the microinjection was in the STN compared with the ZI, suggesting that we cannot exclude the possibility that drug diffusion from the ZI injection site to the STN was responsible for these abnormal movements.

Another technical issue raised by our study concerns the five consecutive injections in the same animal. Yet, although we cannot totally exclude the presence of a lesion, this is unlikely to have influenced our results, because neurons could be recorded at the end of the sessions. Moreover, behaviors were not different between control sessions either side of the bicuculline injections, indicating that the previous injection had no residual effect.

In the present study, bicuculline was used to increase the activity of STN neurons. Electrophysiological studies have shown that bicuculline increases the activity of subthalamic neurons (Féger et al., 1989; Robledo and Féger 1990), but that when the 

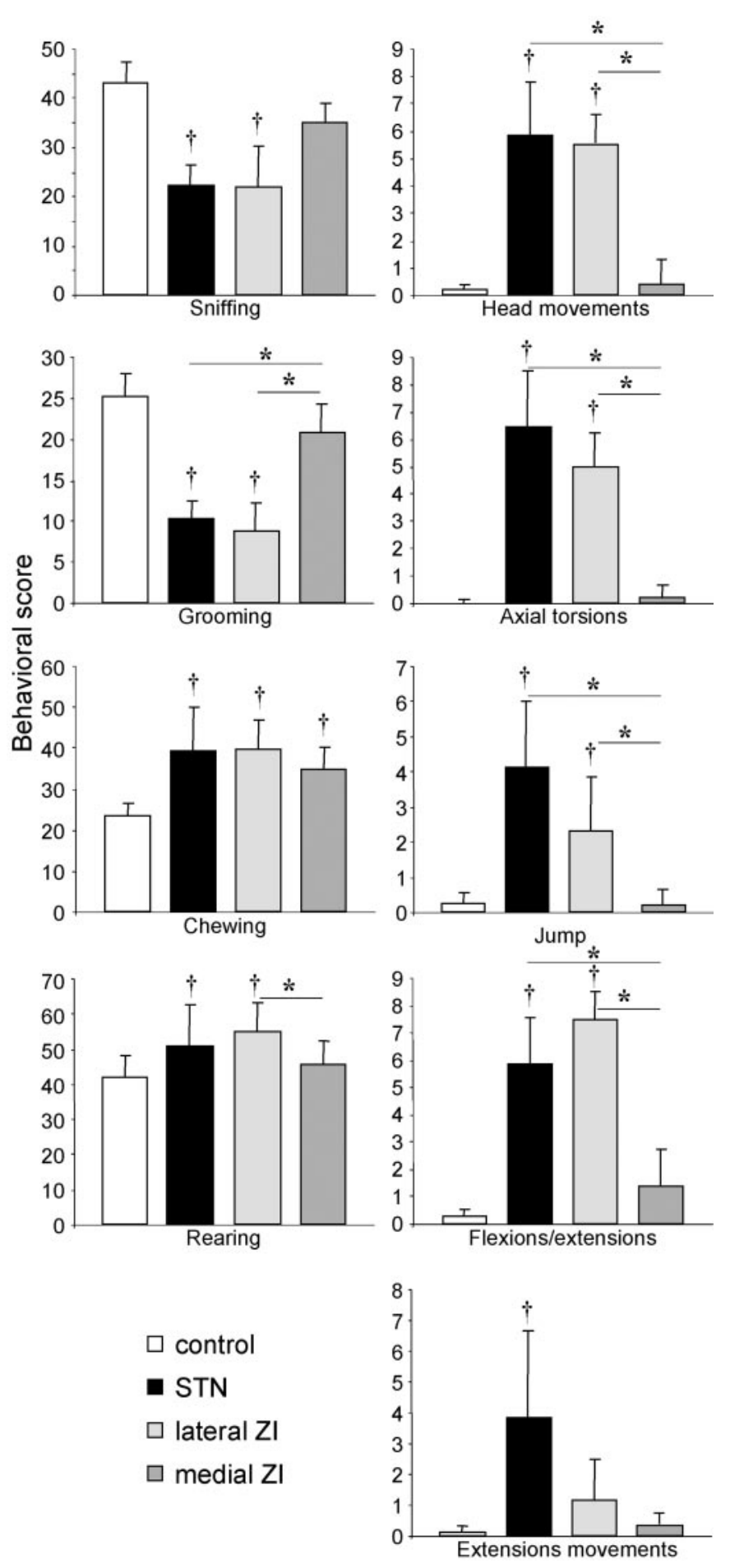

Figure 7. Site specificity of the behavioral changes induced by bicuculline $(100 \mathrm{ng} / \mu \mathrm{l})$. Values represent group mean values \pm SEM of behavioral scores. Dagger indicates significant difference from saline injection (ANOVA and Fisher's post hoc analysis; ${ }^{\dagger} p<0.05$ ); asterisks indicate significant differences among STN, medial ZI, and lateral ZI bicucullineinjected groups (ANOVA and Fisher's post hoc analysis; * $p<0.05$ ).

bicuculline concentration reaches a certain threshold the opposite effect can be observed (Féger et al., 1989). The likely hypothesis for this inhibition is that when subthalamic neurons become too hyperactive they stop discharging, probably because of a depolarization block (Féger et al., 1989; Robledo and Féger, 1990).
However, the concentration of bicuculline used in our study $(0.025-0.1 \mu \mathrm{g} / \mu \mathrm{l})$ was much lower than that required to produce a depolarization block $(0.75 \mu \mathrm{g} / \mu \mathrm{l})$. Indeed, Féger et al. (1989) showed that microinjection of bicuculline into the STN at 0.18 and $0.37 \mu \mathrm{g} / \mu \mathrm{l}$ always induced a significant increase in mean spontaneous neuronal discharge rate and never inhibited neuronal activity. Taken together, these results indicate that the behavioral changes observed in our study were probably due to a hyperactivity of the neurons in the STN or the ZI.

The most surprising result of our study is the absence of parkinsonian signs, such as akinesia and postural rigidity, when hyperactivity of the STN was induced. Indeed, because STN neurons are hyperactive in parkinsonian syndrome after nigrostriatal degeneration, one would expect a hyperactivity of this structure to produce a parkinsonian syndrome. Yet, in our study, this was not the case. Moreover, activation of the ZI induced a locomotor activity and the same kind of behavioral effects as those induced by an activation of the STN. Our results are in agreement with previous studies in various species. (1) Crossman et al. (1984) showed in the normal monkey that bicuculline injections into or close to the STN induced dyskinetic movements typical of hemiballism and that injections of bicuculline in the zona incerta induced torticollis and circling behavior, either in isolation or in addition to contralateral limb dyskinesia; (2) in the cat, bicuculline injection in the STN induced turning behavior and injections in the $\mathrm{ZI}$ resulted in tilting of the head along the longitudinal axis of the body (Murer and Pazo, 1993); and (3) in rat, bicuculline application in the STN resulted in symptoms

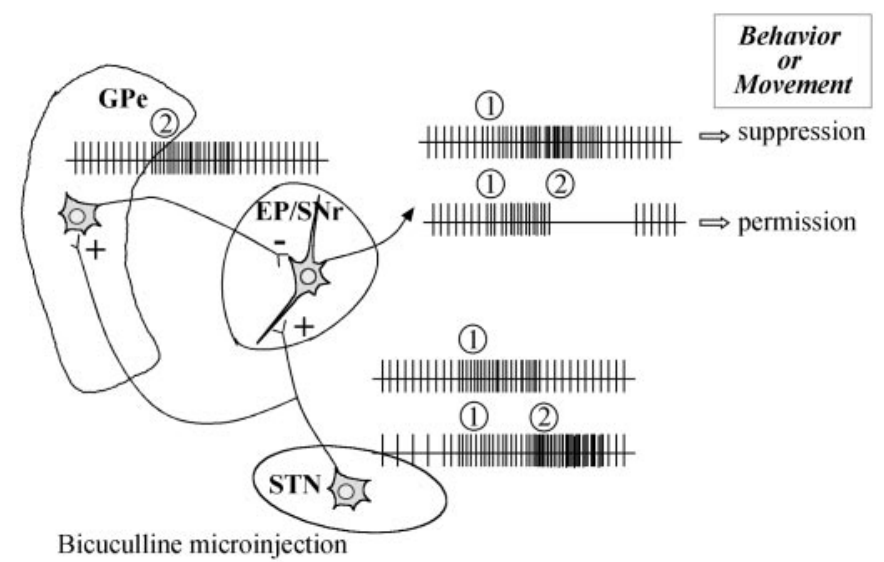

Figure 8. Possible explanation for the consequences of bicuculline microinjection into the STN. (1) Bicuculline microinjection provoked an increase in the neuronal activity of subthalamic neurons, initially leading to an increase in neuronal activity in the output structures of the basal ganglia, which is known to cause behavior suppression. In the rat, STN neurons are highly collateralized, and single STN neurons send axon collaterals to the GP, EP, and SNr. Thus, (2) GP neurons could subsequently display an increase in neuronal activity, leading to an overinhibition of neuronal activity in the output structures of the basal ganglia, eventually enabling behaviors to occur. These consequences may occur during the peak action of bicuculline, $\sim 15$ min after the microinjection. Moreover, it is important to note that the projection from the STN to the $\mathrm{EP} / \mathrm{SNr}$ accounts for $\sim 10 \%$ of the total population of terminals and is evenly spread over perikarya and dendrites (Bevan et al., 1994a,b). In contrast, the projection from the GP to the EP/SNr accounts for $\sim 48 \%$ of the total population of terminals in contact with the perikarya and $5 \%$ of the axodendritic synapses (Bolam and Smith, 1992), and this pallidal influence may be greater than those of the subthalamic nucleus. $E P$, Entopeduncular nucleus; $G P e$, globus pallidus; $S N r$, substantia nigra pars reticulata; $S T N$, subthalamic nucleus. 
resembling seizure manifestations (Scheel-Kruger and Magelund, 1981; Féger et al., 1989; Dybdal and Gale, 2000), which have been interpreted as dyskinesias (Crossman et al., 1984; Féger et al., 1989).

Although these findings are consistent with each other, they are quite unexpected according to the classical model of the basal ganglia circuitry. Such discrepancies with this model have already been described in non-parkinsonian animals regarding the neuronal activity of the substantia nigra pars reticulata. Indeed, Waszczak et al. (2001) showed that microinjection of amphetamine in the ventral striatum induced an increase in locomotor activity that was correlated with an increased firing of the nigral neurons of the pars reticulata. It seems likely that this increase in firing is related to an enhanced activity of the subthalamic neurons.

Another possible explanation for the unexpected consequences of bicuculline injection in the STN may be related to a local effect of the drug, as has already been reported in the pallidum by Matsumura et al. (1995). Indeed, bicuculline may have produced an activation of neurons at the center of injection but a neuronal inhibition at the periphery. Thus, the observed behavioral changes might result from a balance between these two kinds of neuronal activity in both the STN and the ZI, although this has not yet been described for these two structures. Moreover, we showed that behavioral consequences of the microinjection were a reduction of some spontaneous behaviors followed, with a longer latency, by the induction of abnormal movements. These opposite behaviors may involve a balance between a direct activation of the output structures of the basal ganglia [entopeduncular nucleus and substantia nigra pars reticulata $(\mathrm{EP} / \mathrm{SNr})]$ by the STN and an indirect inactivation of the EP/SNr by the globus pallidus (GP), which receives afferent fibers from STN (Deniau et al., 1978; Kita et al., 1983; Parent and Hazrati, 1995), as shown in Figure 8. Finally, another explanation may arise from the localization of the injection site in the different subterritories of the STN. Unfortunately, we were not able to evidence this phenomenon.

As far as the ZI is concerned, it is of interest to note that the most pronounced effects were observed in the lateral ZI and that this part of the ZI displayed a more pronounced hyperactivity in 6-OHDA-lesioned rats (Périer et al., 2000). These data may be explained in part by the known connectivity of the ZI with structures involved in motor activity, such as the superior colliculus (Ricardo, 1981; Roger and Cadusseau, 1985; Ficalora and Mize, 1989), the pedunculopontine nucleus (Kolmac et al., 1998), and the entopeduncular nucleus (Ricardo, 1981). In addition, previous studies have shown that activation of the AMPA subtype of glutamate receptor in the ZI elicits increased locomotor activity (Supko et al., 1991) and that an activation of GABA receptors inhibits locomotor activity. Thus, our results are consistent with these previous findings and suggest that a balanced regulation of glutamate and GABA afferent fibers to ZI neurons is involved in locomotor processes including motor behaviors. Finally, this structure should be taken into account in the treatment of parkinsonian symptoms, because it was recently shown that the best clinical improvement of parkinsonian patients using deep brain stimulation was achieved using electrode poles that were located several millimeters above the STN, corresponding to the pallidothalamic bundle (including Field $\mathrm{H}$ of Forel and the thalamic fascicle), the pallidosubthalamic tract, and/or the zona incerta (Voges et al., 2002).

In summary, our data are in agreement with the notion that the $\mathrm{STN}$ is involved in the pathophysiology of movement disorders, and we have shown that the ZI, and particularly the lateral part, may be implicated in the control of movement, suggesting that the subthalamic region, and not the STN per se, might be involved in the induction of abnormal movements. Nevertheless, it seems that in rats, a unique local hyperactivity of STN neurons is not sufficient to produce parkinsonian symptoms, suggesting that there are certainly major differences between the functioning of these structures in the normal and the pathological state.

\section{REFERENCES}

Baron MS, Wichmann T, Ma D, DeLong MR (2002) Effects of transient focal inactivation of the basal ganglia in parkinsonian primates. J Neurosci 22:592-599.

Benazzouz A, Gross C, Féger J, Boraud T, Bioulac B (1993) Reversal of rigidity and improvement in motor performance by subthalamic highfrequency stimulation in MPTP-treated monkeys. Eur J Neurosci 5:382-389.

Bergman H, Wichmann T, DeLong MR (1990) Reversal of experimental parkinsonism by lesions of the subthalamic nucleus. Science 249:1436-1438.

Bevan MD, Bolam JP, Crossman AR (1994a) Convergent synaptic input from the neostriatum and the subthalamus onto identified nigrothalamic neurons in the rat. Eur J Neurosci 6:320-334.

Bevan MD, Crossman AR, Bolam JP (1994b) Neurons projecting from the entopeduncular nucleus to the thalamus receive convergent synaptic inputs from the subthalamic nucleus and the neostriatum in the rat. Brain Res 659:99-109.

Bolam JP, Smith Y (1992) The striatum and the globus pallidus send convergent synaptic inputs onto single cells in the entopeduncular nucleus of the rat: a double anterograde labelling study combined with postembedding immunocytochemistry for GABA. J Comp Neurol 321:456-476

Carpenter MB, Whittier JR, Mettler FA (1950) Analysis of choreid hyperkinesia in the rhesus monkey. Surgical and pharmacological analysis of hyperkinesia resulting from lesions in the subthalamic nucleus of Luys. J Comp Neurol 92:293-332.

Creese I, Iversen SD (1974) The role of forebrain dopamine systems in amphetamine induced stereotyped behavior in the rat. Psychopharmacologia 39:345-357.

Crossman AR, Sambrook MA, Jackson A (1984) Experimental hemichorea/hemiballismus in the monkey. Studies on the intracerebral site of action in a drug-induced dyskinesia. Brain 107:579-596.

Deniau JM, Hammond C, Riszk A, Féger J (1978) Electrophysiological properties of identified output neurons of the rat substantia nigra (pars compacta and pars reticulata): evidences for the existence of branched neurons. Exp Brain Res 32:409-422.

Dybdal D, Gale K (2000) Postural and anticonvulsant effects of inhibition of the rat subthalamic nucleus. J Neurosci 20:6728-6733.

Féger J, Vezole I, Renwart N, Robledo P (1989) The rat subthalamic nucleus: electrophysiological and behavioural data. In: Neural mechanisms in disorders of movement (Crossman AR, Sambrook MA, eds), pp 37-43. London: J. Libbey.

Ficalora AS, Mize RR (1989) The neurons of the substantia nigra and zona incerta which project to the cat superior colliculus are GABA immunoreactive: a double-label study using GABA immunocytochemistry and lectin retrograde transport. Neuroscience 29:567-581.

Grossman RG (1958) Effects of stimulation of non-specific thalamic system on locomotor movements in cat. J Neurophysiol 21:85-93.

Guridi J, Obeso JA (1997) The role of the subthalamic nucleus in the origin of hemiballism and parkinsonism: new surgical perspectives. Adv Neurol 74:235-247.

Hamada I, DeLong MR (1992) Excitotoxic acid lesions of the primate subthalamic nucleus result in transient dyskinesias of the contralateral limbs. J Neurophysiol 68:1850-1858.

Hammond C, Féger J, Bioulac B, Souteyrand JP (1979) Experimental hemiballism in the monkey produced by unilateral kainic acid lesion, in corpus Luysii. Brain Res 171:577-580.

Kita H, Chang HT, Kitai ST (1983) The morphology of intracellularly labeled rat subthalamic neurons: a light microscopic analysis. J Comp Neurol 215:245-257.

Kolmac CI, Power BD, Mitrofanis J (1998) Patterns of connections between zona incerta and brainstem in rats. J Comp Neurol 396:544-555.

Limousin P, Pollak P, Benazzouz A, Hoffmann D, Le Bas JF, Broussolle E, Perret JE, Benabid AL (1995) Effect of parkinsonian signs and symptoms of bilateral subthalamic nucleus stimulation. Lancet 345:91-95.

Martin JP (1927) Hemichorea resulting from a focal lesion of the brain. (The syndrome of the body of Luys.). Brain 50:637-651.

Matsumura M, Tremblay L, Richard H, Filion M (1995) Activity of pallidal neurons in the monkey during dyskinesia induced by injection of bicuculline in the external pallidum. Neuroscience 65:59-70.

Milner KL, Mogenson GJ (1988) Electrical and chemical activation of 
the mesencephalic and subthalamic locomotor regions in freely moving rats. Brain Res 452:273-285.

Murer MG, Pazo JH (1993) Circling behaviour induced by activation of $\mathrm{GABA}_{\mathrm{A}}$ receptors in the subthalamic nucleus. NeuroReport 4:1219-1222.

Parent A, Hazrati LN (1995) Functional anatomy of the basal ganglia 2. The place of subthalamic nucleus and external pallidum in basal ganglia circuitry. Brain Res Rev 20:128-154.

Paxinos G, Watson C (1986) The rat brain in stereotaxic coordinates. Sydney: Academic.

Périer C, Vila M, Féger J, Agid Y, Hirsch EC (2000) Functional activity of zona incerta neurons is altered after nigrostriatal denervation in hemiparkinsonian rats. Exp Neurol 162:215-224.

Ricardo JA (1981) Efferent connections of the subthalamic region in the rat. II. The zona incerta. Brain Res 214:43-60.

Robledo P, Féger J (1990) Excitatory influence of rat subthalamic nucleus to substantia nigra pars reticulata and the pallidal complex: electrophysiological data. Brain Res 518:47-54.

Roger M, Cadusseau J (1985) Afferents to the zona incerta in the rat: a combined retrograde and anterograde study. J Comp Neurol 241:480-492.

Romanowski CA, Mitchell IJ, Crossman AR (1985) The organisation of the efferent projections of the zona incerta. J Anat 143:75-95.

Scheel-Kruger J, Magelund G (1981) GABA in the entopeduncular nu- cleus and the subthalamic nucleus participates in mediating dopaminergic striatal output functions. Life Sci 29:1555-1562.

Supko DE, Uretsky NJ, Wallace LJ (1991) Activation of AMPA/kainic acid glutamate receptors in the zona incerta stimulates locomotor activity. Brain Res 564:159-163.

Vila M, Périer C, Féger J, Yelnik J, Faucheux B, Ruberg M, RaismanVozari R, Agid Y, Hirsch EC (2000) Evolution of changes in neuronal activity in the subthalamic nucleus of rats with unilateral lesion of the substantia nigra assessed by metabolic and electrophysiological measurements. Eur J Neurosci 12:337-344.

Voges J, Volkmann J, Allert N, Lehrke R, Koulousakis A, Freund HJ, Sturm V (2002) Bilateral high-frequency stimulation in the subthalamic nucleus for the treatment of Parkinson disease: correlation of therapeutic effect with anatomical electrode position. J Neurosurg 96:269-279.

Waszczak BL, Martin L, Boucher N, Zahr N, Sikes RW, Stellar JR (2001) Electrophysiological and behavioral output of the rat basal ganglia after intrastriatal infusion of D-amphetamine: lack of support for the basal ganglia model. Brain Res 920:170-182.

Whittier JR, Mettler FA (1949) Studies on the subthalamus of the rhesus monkey. II. Hyperkinesia and other physiologic effects of subthalamic lesions, with special reference to the subthalamic nucleus of Luys. J Comp Neurol 90:319-372. 